\title{
Iterative Channel Estimation For The Chinese Digital Television Terrestrial Broadcasting Systems With The Multiple-antenna Receivers
}

\author{
Zi-Wei Zheng \\ ${ }^{1}$ College of Information Science and Engineering, Ningbo University, 315211 Ningbo, P. R. China \\ ${ }^{2}$ College of Information Engineering, Dalian Maritime University, 116026 Dalian, P. R. China \\ E-mail: ziwei_zheng@yahoo.com.cn
}

Keywords: Channel estimation; digital television terrestrial broadcasting system; multiple-antenna receivers.

\begin{abstract}
Orthogonal frequency division multiplexing is an effective against multipath fading and high data throughput wireless channel transmission technology. Assistance with the inverse fast Fourier transform and fast Fourier transform operation, orthogonal frequency division multiplexing modulation and demodulation operations of the system convenient and convenient hardware implementation, orthogonal frequency division multiplexing, so in the modern digital television terrestrial broadcasting the system is widely used to support high performance bandwidth-efficient multimedia services. Broadband multi-carrier orthogonal frequency division multiplexing with multi-antenna and multi-antenna receiving system, to increase the diversity gain and improve the capacity of the system in different multipath fading channel. Accurate channel estimation in a simple channel equalization and decoding of broadband multi-carrier orthogonal frequency division multiple-antenna receiver and channel estimation accuracy and multiplexing system is very important, is the key to the performance of the overall broadband multi-carrier orthogonal frequency division multiplexing system in the multi-antenna receiver bit error rate. In this paper, iterative channel estimation to plan for digital terrestrial television broadcasting broadband multi-carrier orthogonal frequency division multiple antenna receiver multiplexing system proposal.
\end{abstract}

\section{Introduction}

Faced with the challenges of modern communication, high data throughput requirements, extensive research has been paid to broadband modulation. The multi-carrier modulation is one of the most important wide band modulation techniques. Orthogonal frequency division multiplexing (OFDM) technology, a specific multi-carrier modulation, has become a popular technique to combat ISI (Inter Symbol Interference) channel. ISI is to avoid insert intervals defender. Forward error correction (FEC) technology through a simple channel equalization and the former, to overcome the effects of selective fading [1]. With the aid of inverse fast Fourier transform and fast Fourier transform operations, both modulation and demodulation operations of the OFDM systems facilitate convenient hardware implementations, and thus make the OFDM to be extensively used in broadcasting domain, such as the Digital Video Broadcasting for Terrestrial Television (DVB-T) proposed as the European digital television standard [2], and the Terrestrial Digital Multimedia/ Television Broadcasting DMB-T [3] proposed by Tsinghua University for the digital television terrestrial broadcasting standard of the People's Republic of China. When delay spread is longer than the length of guard intervals, the performance of OFDM is lower sharply [1]. To cope with the long multipath propagation in the digital television terrestrial broadcasting, long guard intervals may be introduced with the cost of lower effective data rate, or long OFDM symbol can be use to save the data rate but at the expense of implementation complexity and long decoding delay. Therefore, the length of the guard intervals and the length of the OFDM symbol must be selected tradeoff between system performance and the practical implementation complexity. In DVB-T, the CP, as guard interval and course symbol synchronization, is introduced to cope with multipath signals. Furthermore, DVB-T 
sends a large amount of training symbols (more than $10 \%$ of the data symbols) in order to facilitate the fine symbol synchronization and channel estimation [2]. In the Chinese digital television terrestrial broadcasting system DMB-T, instead of CPs, time domain synchronous OFDM (TDS-OFDM) inserts pseudo-random number (PN) sequences as the guard intervals, which also serve for synchronization and channel estimation [3]. After removing the PN sequences at the receiver, TDS-OFDM is equivalent to the zero-padded OFDM (ZP-OFDM) [4]. In [4], Muquet et al. proposed a low complexity equalizer for ZP-OFDM, compared ZP-OFDM and CP-OFDM, and numerically showed that ZP-OFDM with the equalizer outperformed CP-OFDM in terms of bit error rates, both in coded and uncoded cases. The reasonable time domain combination of the guard intervals and the training symbols reduces transmission overhead and provides a better channel spectral efficiency performance [3]. The separation of training and data makes the synchronization independent on the specific modulation type for the useful data and increases the modularity of the entire system. The technique of multiple antennas at the transmitter and the receiver (MIMO) is well documented, where each pair of transmitter and receiver antennas provides a different signal path from the transmitter to the receiver, and multiple independently faded replicas of the data symbol can be obtained at the receiver. The multiple-antenna broadband multi-carrier orthogonal frequency division multiplexing systems increase diversity gain and enhance system capacity on a time varying multipath fading channel. It is known that multiple-antenna OFDM systems are very sensitive to the channel estimation error and phase noise [1]. Accurate channel estimation is important for the application of simple channel equalization for the multiple-antenna broadband multi-carrier OFDM systems and the accuracy of the channel estimation is crucial to the performance of the overall multiple-antenna OFDM system in terms of the symbol error rate (SER), including the multiple-antenna OFDM-based digital television terrestrial broadcasting (DTTB) systems [1]. Therefore, novel schemes should be proposed to deal with the problem of channel estimation for the multiple-antenna OFDM-based systems under different assumptions. In this paper, a channel estimation scheme is proposed for the multiple-antenna TDS-OFDM-based Chinese digital television terrestrial broadcasting system.

\section{Problem Representation}

The Signal Frame consists of two parts, the Frame Head and the Frame Body, which is shown in figure 1. The Frame Head consists of a pre-amble, a PN sequence with 255 symbols, and a post-amble. The pre-amble and post-amble are cyclical extensions of the PN sequence, the length of pre-amble ${ }^{L_{\text {pre }}}$ can be defined as 24, 25, and 50, and the post-amble ${ }^{L_{p o s t}}$ can be defined as 25,104 , and 115, dependent on the maximum delay spread of the channel. In the simulation below, the pre-able ${ }^{L_{\text {pre }}}$ has 50 symbols, and post-amble ${ }^{L_{\text {post }}}$ has 115 symbols, total guard interval length $N_{t}$ is 420 symbols. BPSK modulation is used in the Frame Head for robust synchronization and channel estimation. The cyclical extensions of the PN sequence used for the pre-amble and post-amble can be used for quick PN acquisition. Since the cyclical extensions are known at the receiver, they can easily be suppressed by using simple operation. The Frame Body has 3780 symbols, 3744 symbols carried the data and 36 symbols carried the Transmission Parameter Signaling (TPS). OFDM modulation scheme is used for Frame Body. Some information symbols are set null. In the simulations of this paper, it is assumed that twenty information symbols are set null, which hardly have an effect on the spectral efficiency performance of the system. The TPSs carry information for the demodulator to automatically adapt to the incoming transmission such as: FEC inner code rate, time interleaver length etc. The sub-carrier position and the symbol information of part of the TPSs are known at the receiver, which are proposed for tracking the common phase rotation (CPR), i.e., the phase rotation of the desired sample (part effects of the phase noise). 
At the transmitter side of the Chinese digital television terrestrial broadcasting system, each 3780 of the mapping symbols are grouped into one OFDM frame body. Information symbols $\mathbf{s}(k)=\left[s_{0}(k), s_{1}(k), \cdots, s_{N_{s}-1}(k)\right]^{T} \quad\left(N_{s}=3780\right)$ are assigned to every specific OFDM data frame body by the inverse fast Fourier transform (IFFT) block. PN sequence with pre-amble and post-amble, $\mathbf{t}(k)=\left[t_{0}(k), t_{1}(k), \cdots, t_{N_{t}-1}(k)\right]^{T}$, is inserted for synchronization and channel distortion evaluation in front of each OFDM frame body. A square root raised cosine (SRRC) pulse shaping is performed to limit the transmitted signals to $8 \mathrm{MHz}$ bandwidth before they are up-converted and power amplified and transmitted over the channel. The symbol rate is same for both the Frame Head and the Frame Body, which is 7.56 MSPS.

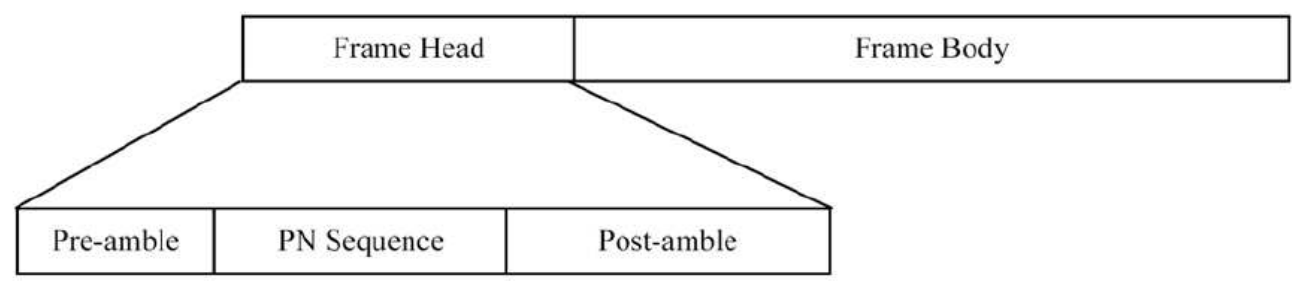

Fig. 1. Signal frame format for the Chinese digital television terrestrial broadcasting systems with the multiple-antenna receiver

At the receiver side of the multiple-antenna Chinese digital television terrestrial broadcasting system, the received signals are down-converted, SRRC pulse shaping, sampled. Then, the samples are fed in parallel to the timing estimator, the frequency offset estimator, the channel estimator, and the fast Fourier transform (FFT) block, respectively. A PN sequence, the same as the frame header, is generated by the local PN code generator. With the PN sequence, the timing and frequency offset estimation are performed. Also, the PN sequence is used as the channel sounding signal.

Suitable designed pre-amble symbols are used to make the PN sequence not be affected by the previous frame due to multipath distortion. Suitable designed post-amble symbols are used to make the OFDM symbols not be affected by the PN sequence due to multipath distortion. Assumed that the pre-amble is with ${ }^{L_{\text {pre }}}$ symbols length, the PN sequence is with $N^{\mathrm{PN}}$ symbols length, the post-amble is with ${ }^{L_{\text {post }}}$ symbols length, and the OFDM Frame Body block is with $N$ symbols length. Assumed that the transmitted signal $S(n)$ is passing through a multipath channel $h(n) . h(n)$ is the composite channel response, taking into account the effects of transmitter filter, multipath channel, and receiver filter. At the reception side, multiple antennas are used. It is assumed that the separations between each antenna are of one half wavelength or more, thus make the signals received from spatially separated antennas having essentially uncorrelated envelopes. At every antenna, the local oscillator down-converts and transforms the RF signals to baseband, then, the baseband signals are passing through a lowpass filter $p(t)$, an anolog to discrete $(A / D)$ converter with sampling period $T$, a serial to parallel $(S / P)$ converter, an cyclic prefix remover, the unit norm fast Fourier transform (FFT) $\mathbf{F}$ and the reverse mapping $\Pi(k)^{T}$ operators. Finally, multiple-antenna diversity reception is performed.

In the presence of phase noise, after completion of automatic gain control (AGC), signal detection, timing and frequency offset correction, the received signal $r(n)$ at the ${ }^{a_{r} \text { th }}$ receiving antenna can be formulated as

$$
r_{a_{r}}(n)=S(n) \otimes h_{a_{r}}(n) \cdot e^{j \theta_{a_{r}}^{\mathrm{PHN}}(n)}+\xi_{a_{r}}(n)
$$

where $\otimes$ denotes convolution, ${ }_{a_{r}}{ }^{\text {PHN }}(n)$ indicates the phase noise, $\xi_{a_{r}}(n)$ indicates AWGN.

The correlation function

$$
R^{r_{a_{r}}{ }^{\mathrm{PN}}}(n)=\frac{1}{N_{\mathrm{PN}}} \sum_{i=0}^{N_{\mathrm{PN}}-1} r_{a_{r}}(i-n) \cdot S^{\mathrm{PN}}(i)
$$

between the received signal ${ }^{r_{r}}(n)$ and the locally generated PN sequence $S^{\mathrm{PN}}$ is 


$$
\begin{aligned}
& \qquad R^{r_{a_{r}}^{\mathrm{PN}}}(n)=R^{S S^{\mathrm{PN}}}(n) \otimes h_{a_{r}}(n) \cdot e^{j \theta_{a_{r}}^{\mathrm{PHN}}(n)}+\xi_{a_{r}}(n) \\
& \text { where } R^{S S^{\mathrm{PN}}}(n)=\frac{1}{N^{\mathrm{PN}}} \sum_{i=0}^{N^{\mathrm{PN}}-1} S(i-n) \cdot S^{\mathrm{PN}^{*}}(i) \text { denotes the correlation between the signal } S \text { and } S^{\mathrm{PN}},(\cdot)^{*} \\
& \text { denotes the complex conjugate. } \\
& \text { In an idea case, during every one period of the PN sequence, there exists } \\
& \qquad R^{S S^{\mathrm{PN}}(n)=} \\
& \qquad \begin{array}{ll}
R^{S^{\mathrm{PN}} S^{\mathrm{PN}}}=\frac{1}{N^{\mathrm{PN}}} \sum_{i=0}^{\mathrm{PN}^{\mathrm{PN}}-1} S^{\mathrm{PN}}(i) \cdot S^{\mathrm{PN}}(i) & n=0 \\
\xi^{\prime}(n) & n \neq 0
\end{array}
\end{aligned}
$$

where $R^{S^{\mathrm{PN}} S^{\mathrm{pN}}}$ is known at the receiver side, $\xi^{\prime}(n)$ is a complex Gaussian noise with variance of $\left(R^{S^{\mathrm{PN}} S^{\mathrm{PN}}}\right)^{2} / N^{\mathrm{PN}}$.

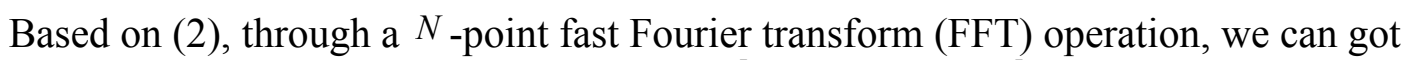

$$
\begin{aligned}
& \operatorname{FFT}\left(R^{a_{a_{r}} S^{\mathrm{pN}}}(n)\right)=\operatorname{FFT}\left(R^{S \mathrm{P}^{\mathrm{NN}}}(n)\right) \cdot\left[H_{a_{r}}(f) \cdot e^{j \theta_{a_{r}}{ }^{\mathrm{pHN}}(n)}(f)\right]+\xi_{a_{r}}(f) \\
& =\operatorname{FFT}\left(R^{S S^{\mathrm{pN}}}(n)\right) \cdot H_{a_{r e f}}(f)+\xi_{a_{r}}(f)
\end{aligned}
$$

where $\operatorname{FFT}(\cdot)$ denotes the FFT operation, $H_{a_{r}}(f)$ is the actual channel frequency response, $H_{a_{r e f}}(f)$ is the effective composite channel frequency response including the actual channel frequency

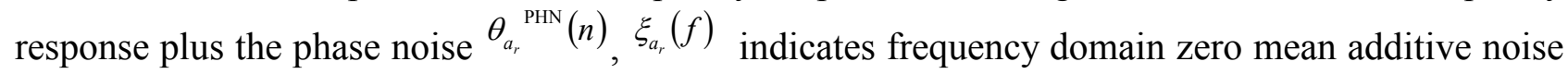
at the ${ }^{a_{r} \text { th }}$ antenna.

Denoted $H_{a_{r e f} k}$ as the effective composite channel frequency response at the $k$ th sub-carrier at the $a_{r}$ th antenna and it can be written as

$$
H_{a_{r e f}}=e^{j \theta_{a_{r}}^{\mathrm{PHN}}(n)} \cdot \sum_{l=0}^{N_{h}} h_{l} e^{-j 2 \pi k \tau_{l} f_{k}}
$$

where $N_{h}$ is the channel length, $\left\{\tau_{l}\right\}$ are the path time delays, $f_{\Delta}$ is the sub-carrier spacing, $f_{k}=k f_{\Delta}$ is the sub-carrier frequency of the $k$ th sub-carrier at the $a_{r}$ th antenna.

From (4), the channel estimation at the $k$ th sub-carrier at the ${ }^{a_{r}}$ th receiving antenna is easily got as

$$
\hat{H}_{a_{r e f}}=\left[\operatorname{FFT}\left(R^{S S^{\mathrm{PN}}}(n)\right)\right]_{k}^{-1} \cdot\left[\operatorname{FFT}\left(R^{r_{a r} r^{\mathrm{PN}}}(n)\right)\right]_{k}
$$

where $n$ is the time index, $k \in\{0,1, \cdots, N\}$ is the sub-carrier (frequency) index.

To further improve the channel estimation performance, an iterative algorithm is developed based on the expectation-maximization (EM) algorithm [5] for each receiving antenna at the receiver of the Chinese digital television terrestrial broadcasting systems. The total noise variance is estimated by using those null symbols according to the signal frame format for the Chinese digital television terrestrial broadcasting systems with the multiple-antenna receiver [3]. Then, combined with all antennas of the receiver, from the PN sequence of the frame head, based on the above channel estimation method, the channel estimation of the composite channel frequency response of the data carrying part Frame Body at the receiver can be got.

The Chinese digital television terrestrial broadcasting system with parameters as shown in Table I are used for simulations to verify the efficiency of the proposed channel estimation scheme. Meanwhile, specially scattered twenty information symbols are set null, and sixteen TPSs are known at the receiver. The simulation channels are the DVB-T Fix Reception F1 version channel model [2], the 6-path typical hilly terrain channels with parameters randomly generated from COST 207 specifications [6] under different maximal Doppler frequencies, $f_{d}=10 \mathrm{~Hz}$, which corresponds to receiver velocity of 12.5 to $23 \mathrm{~km} / \mathrm{h}$ in the TV UHF band $(@ 470 \ldots 862 \mathrm{MHz})$, and $f_{d}=60 \mathrm{~Hz}$, which corresponds to receiver velocity of 75 to $138 \mathrm{~km} / \mathrm{h}$ in the TV UHF band (@470...862MHz). Local 
oscillator crystal offset normalized the sampling period is $10 \mathrm{ppm}$, carrier-frequency offset is of 3 -subcarrier spacing. It is also assumed that the separations between each antenna are of one half wavelengths or more, and that the signals received from spatially separated antennas have essentially uncorrelated envelopes. Figure 2 shows the mean squared error (MSE) performance of the proposed channel estimation scheme for different radio channel environments with different number of receiver antennas. The mean square error (MSE) values between the transfer function of transmission channel $H$ and its estimate $\hat{H}$ can be expressed and calculated as $\operatorname{MSE}=E\left[\|H-\hat{H}\|^{2}\right]$, where $E[x]$ is ensemble average of a random sequence $x$. From figure 2, we can see that the proposed channel estimation scheme achieve perfect MSE performance under different channel situations.

Table 1. Parameters of the Chinese DTTB systems

\begin{tabular}{c|c}
\hline Channel bandwidths & $8 \mathrm{MHz}$ \\
\hline Occupied bandwidths & $7.56 \mathrm{MHz}$ \\
\hline $\begin{array}{c}\text { Square Root Raised Cosine } \\
\text { Pulse shaping filter }\end{array}$ & alpha $=0.05$ \\
\hline Sampling rate & $30.4 \mathrm{MSPS}$ \\
\hline Symbols per Frame Body & 3780 \\
(FFT size) & 3744 (FEC) +36 (TPS) \\
\hline Frame Body Period & $500 \mu \mathrm{s}$ \\
\hline Sub-carrier spacing & $2.0 \mathrm{kHz}$ \\
\hline Frame Body & QPSK, 16QAM, 64QAM \\
\hline Sub-carrier modulation & each OFDM frame \\
\hline TPS frequency & 36 symbols (72 bits) \\
\hline TPS length & QPSK \\
\hline TPS modulation & 420 symbols \\
$(1 / 9$ of 3780) \\
\hline Frame Head duration & 255 \\
\hline PN sequence length & 50 \\
\hline Pre-amble length & 115 \\
\hline Post-amble length &
\end{tabular}

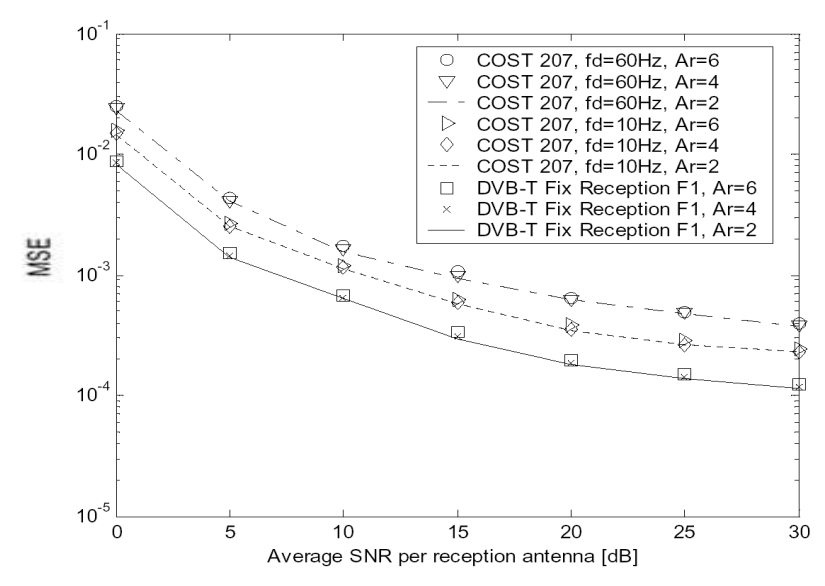

Fig. 2. MSE performance of the proposed channel estimation scheme under different channel situations

\section{Summary}

An iterative channel estimation scheme is proposed for the multi-carrier Chinese digital television terrestrial broadcasting systems with the multiple-antenna receiver.

\section{Acknowledgments}

This work was supported in part by the National Science Foundation of China (No. 60972063), the National Science and Technology Major Project (No. 2011ZX03002-004-02), the Science Foundation of Zhejiang Province (No. R1110416), the Specialized Research Fund for the Doctoral Program of Higher Education (No. 20113305110002), the Program for Technology Innovation Team of Ningbo Government (No. 2011B81002). 


\section{References}

[1] G. L. Stuber, J. R. Barry, S. W. McLaughlin, Y. G. Li, M. A. Ingram, and T. G. Pratt, "Broadband MIMO-OFDM wireless communications,” Proc. IEEE, vol. 92, no. 2, pp. 271-294, Feb. 2004.

[2] ETSI EN 300744 V1.4.1, Digital Video Broadcasting (DVB); Frame structure, channel coding and modulation for digital terrestrial television, Jan. 2001.

[3] GB 20600-2006, National Standard of P. R. China; Frame structure, channel coding and modulation for digital terrestrial television, Aug. 2006.

[4] B. Muquet, Z. Wang, G.B. Giannakis, M. de Courville, and P. Duhamel, "Cyclic-Prefixed or Zero-Padded Multicarrier Transmissions?,” IEEE Trans. Commun., vol. 50, pp. 2136 -2148, Dec. 2002.

[5] J. Choi. Adaptive and Iterative Signal Processing in Communications. Cambridge, U.K.: Cambridge University Press, 2006.

[6] M. Failli, "Digital land mobile radio communications," CIC Inf. Technol. and Sciences, Brussels, Belgium, COST 207 Final Rep., 1989, pp. 135-166. 